\title{
THE NEOSHIELD PROJECT FOR NEAR-EARTH OBJECT IMPACT THREAT MITIGATION
}

International Seminar on Nuclear War and Planetary Emergencies - 48th Session

By Line Drube*, Alan Harris* and the NEOShield Consortium

* Institute of Planetary Research of the German Aerospace Center (DLR), Berlin.

\section{Introduction}

Every day the Earth collects some 100 tonnes of cosmic material, most of it in the form of dust or small rocks, which burn up as meteors in the atmosphere. Sometimes, however, larger objects, asteroids or comets, enter the Earth's atmosphere, which can cause considerable damage. The object that exploded over the Russian city of Chelyabinsk in February 2013 had a diameter of only 17-20 m, yet it produced a blast wave that damaged more than 7000 buildings and some 1600 people sought medical help for injuries (see Figure 1). The Chelyabinsk asteroid entered the Earth's atmosphere with a velocity of 65000 $\mathrm{km} / \mathrm{h}$ and, due to the heating and stresses caused by compression of the air, exploded at an altitude of some $25 \mathrm{~km}$ releasing energy 30 times that of the Hiroshima bomb. The potentially devastating effects on Earth of a collision with a large asteroid or comet are now well recognized by scientists. So the question is now, can we protect our civilization from the next major impact?

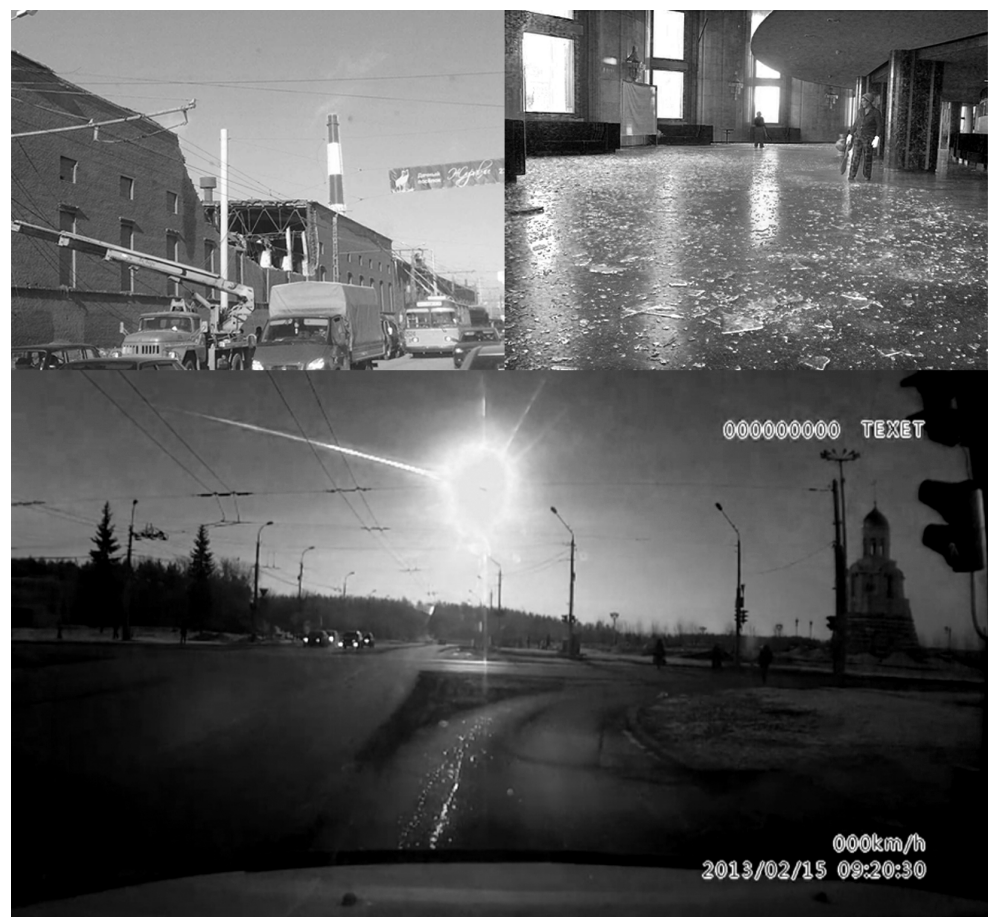

Figure 1: Collage of images from the Chelyabinsk impact. Top row: a collapsed roof of a building and the broken windows of another are both examples of damage caused by pressure waves from the airburst. Bottom row: the spectacular explosive disintegration of the incoming asteroid. Images from Wikipedia. 
An indication that awareness of the impact hazard has reached political circles in Europe was the call for proposals for research projects to address scientific and technical aspects of the impact hazard issued by the European Commission's Seventh Framework Programme in 2010. The NEOShield project, a consortium of 13 partners from 6 countries, won a total of 5.8 million euros funding from January 2012 until May 2015 to investigate mitigation-relevant NEO physical properties and potential mitigation techniques (Harris et al., 2013). The consortium consisted of researchers and engineers from the German Aerospace Centre DLR (the project coordinator), Paris Observatory, French National Centre for Scientific Research, The Open University (United Kingdom), Fraunhofer Ernst-Mach Institute (Germany), Queen's University Belfast (United Kingdom), Astrium (now Airbus Defence and Space) in Germany, UK, and France, Deimos Space (Spain), SETI Institute (USA), Russian Federal Space Agency's TsNIIMash Institute, and The University of Surrey (United Kingdom).

We present a brief overview of the NEOShield project in terms of the four main themes addressed.

\section{Mitigation demonstration mission designs}

NEOShield paved the way for future demonstration missions by providing detailed designs of technically and financially realistic space missions to demonstrate the effectiveness of mitigation techniques. The NEOShield technical partners, with the support of the science team, designed three feasible mission concepts, that will facilitate the rapid development of actual test missions at a later stage, whenever the requisite funding becomes available. The three deflection techniques considered are:

- The kinetic impactor, in which a spacecraft transfers momentum to an asteroid by impacting it at a very high velocity.

- Blast deflection, in which an explosive, such as a nuclear device, is detonated near, on, or just beneath the surface of the object.

- The gravity tractor, in which a spacecraft hovering under power in close proximity to an asteroid uses the gravitational force between the asteroid and itself to tow the asteroid onto a safe trajectory relative to the Earth ("slow-pull" method).

The above three methods are currently considered the most realistic techniques, depending on the asteroid size and the length of time available for the mitigation attempt (see Figure 2).

The NEOShield work also included the compilation of a prioritized list of accessible and realistic asteroid targets for test missions (Figure 3) and the corresponding mission trajectory calculations. 


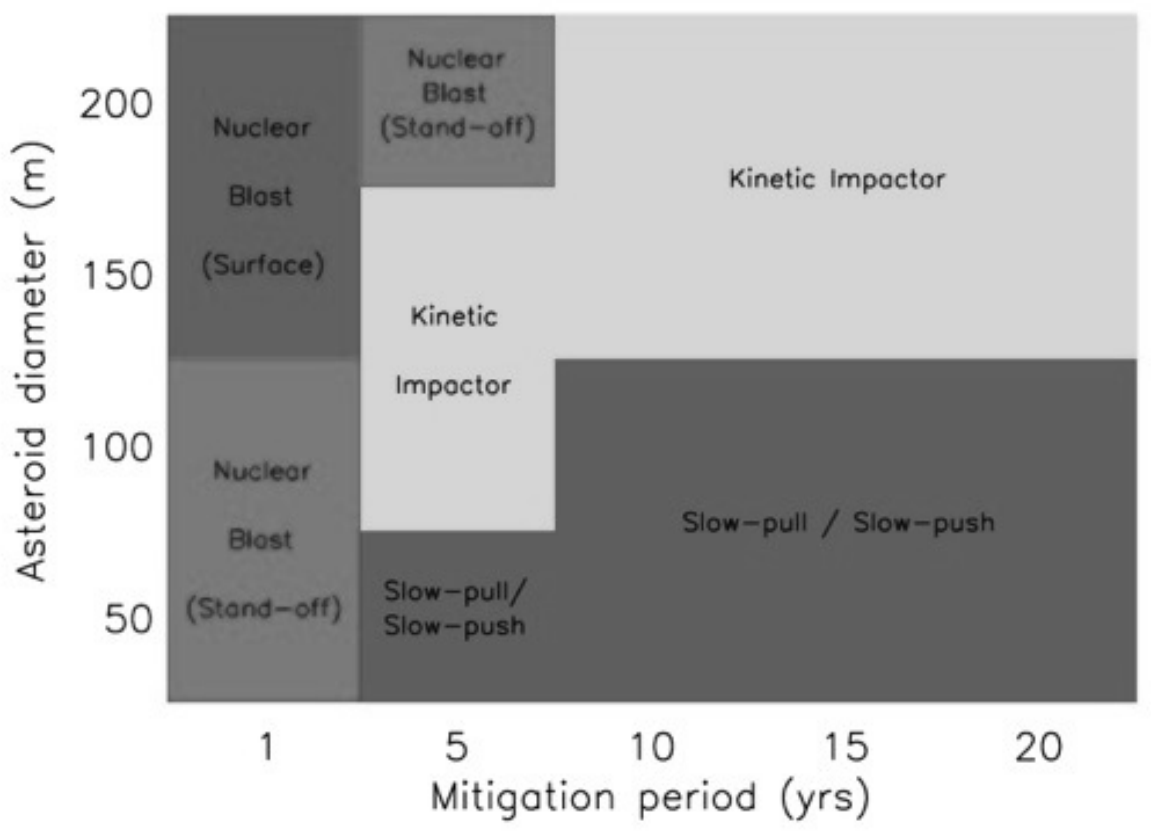

Figure 2: The results of a NEOShield trade-off study by Airbus Defence and Space (UK), showing the best choice of mitigation methods for different asteroid diameters and the time available for the mitigation attempt. For asteroids smaller than 50 meters, an evacuation of the impact area is judged more appropriate than a deflection attempt. Figure credit: S. Eckersley (NEOShield, Airbus Defence and Space Limited, UK) and D. Perna (NEOShield, Observatoire de Paris, LESIA). (Drube et al, 2014)

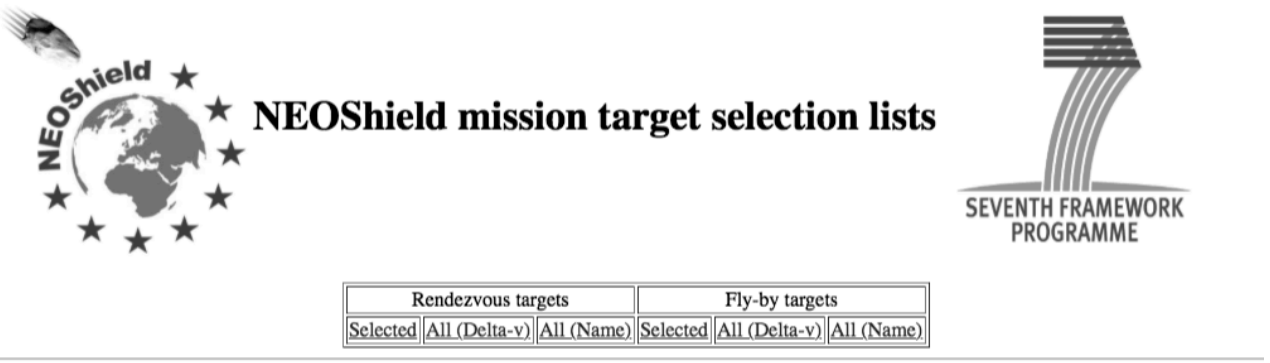

\section{All NEOs with Rendezvous delta-v $<5 \mathrm{~km} / \mathrm{sec}$, ordered by delta-v}

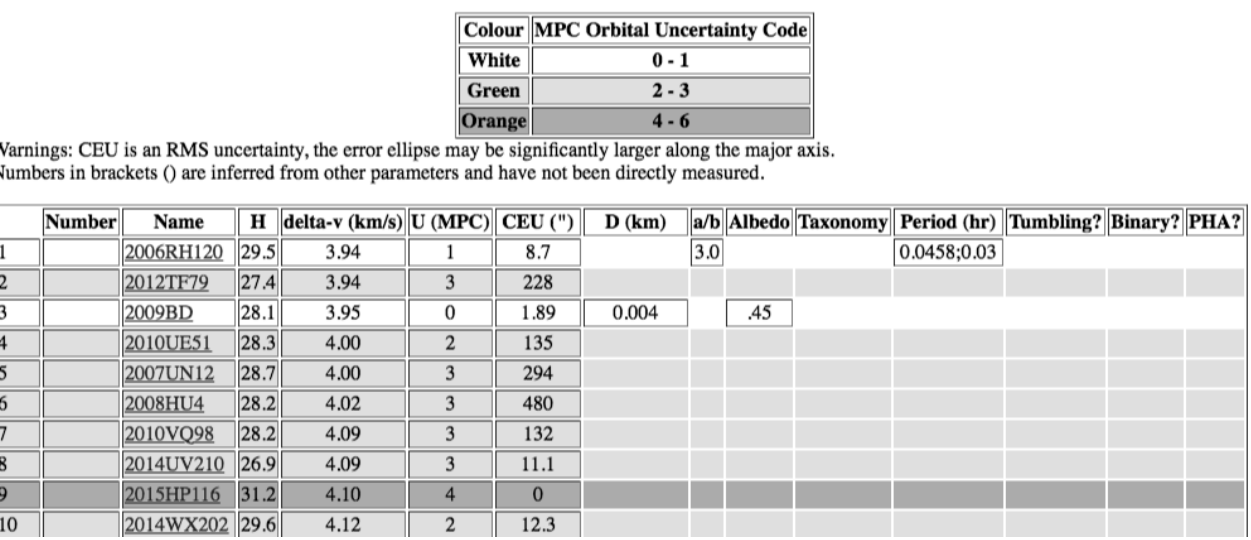

Figure 3: A screen shot of the start of the continuously updated list of potential target asteroids for deflection test missions, listed in order of increasing delta-v (a measure of the accessibility of the asteroid). The list, maintained by Alan Fitzsimmons of Queen's University Belfast, can be found on: http://star.pst.qub.ac.uk/\%7Eaf/lowdv_neos/ 


\section{Technology development}

Further development of some key technologies is a prerequisite to carrying out a deflection test on a small asteroid, such as improvements to spacecraft guidance, navigation and control systems.

In the case of the blast deflection, NEOShield work concentrated on theoretical studies of the effects of a nuclear explosion on a typical small asteroid (Meshcheryakov and Lipnitskii, 2015).

Other NEOShield results include solutions to issues concerning the operation of gravity tractor and kinetic impactor NEO deflection missions (Ummen and Lappas, 2014; Chapuy et al., 2014, respectively), and a discussion of the importance of high precision astrometry of NEOs for asteroid threat assessment and mitigation (Eggl et al., 2013).

\section{Mitigation-relevant physical properties of asteroids}

Project partners also carried out research into the mitigation-relevant physical properties of NEOs, including the analysis of available observational data, laboratory experiments on asteroid analog materials, and computer modelling and impact simulations. The aim of the scientific work was to facilitate predictions of the outcome of deflection attempts using different techniques on a variety of NEO types.

The main objective of the analysis of the observational data was to investigate the most likely properties of future potential impactors that could trigger a space-borne mitigation action. By narrowing the range of expected properties (e.g. albedo, shape, spin rate, mineralogy, internal structure), a rational basis for the choice of objects to serve as targets in deflection test missions can be provided.

In the course of the work a new method was discovered for helping to identify metal-rich asteroids. Observations with infrared telescopes have revealed that the surfaces of some asteroids appear cooler than others at similar distances from the Sun. The reason appears to be related to the thermal conductivity of the asteroid material: Asteroids orbit the Sun absorbing incident sunlight which they re-emit as heat radiation in the infrared part of the spectrum. A rotating asteroid with a high thermal conductivity, e.g. due to a metal-rich surface, will absorb solar energy deeper into its surface and re-emit some of it as thermal-infrared radiation with a time delay on the night side of the object. Due to the conservation of energy, the energy re-emitted on the day side is reduced and the temperature is consequently lower. By comparing the emitted heat radiation with radar reflectance measurements, we have established that the lower temperatures of many asteroids are due to their high metal content (see Figure 4). The relevance of this result to mitigation derives from the fact that metallic asteroids are denser and presumably much more robust than other asteroid types and can therefore penetrate deeper into the atmosphere and cause more 
damage. The Tunguska airburst, which flattened some $2000 \mathrm{~km}^{2}$ of forest in Siberia in 1908, is attributed to a stony object with a diameter in the range $30 \mathrm{~m}$ - $50 \mathrm{~m}$, whereas a similarly sized metallic asteroid is thought to be responsible for hitting the ground and forming the $1.2 \mathrm{~km}$ - diameter Barringer crater in Arizona. In a heavily populated region a crater-forming event would presumably cause much greater devastation than an airburst. Metal-rich asteroids may differ significantly from their metal-poor stony or carbonaceous counterparts in terms of strength, density, and internal structure, characteristics that could seriously affect the outcome of a deflection attempt. Knowledge of the mineralogy of a threatening object would therefore be of vital importance to mitigation planners.

a
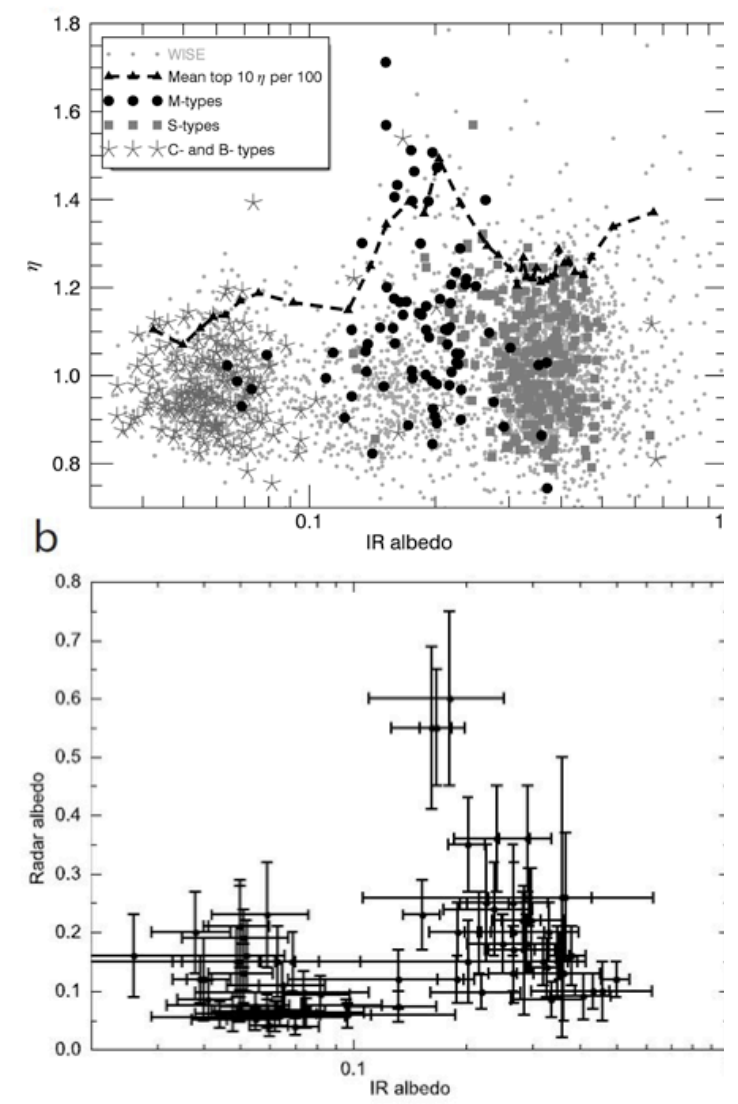

Figure 4:(a) $\eta$ values versus infrared albedo ( $\eta$ is a fitting parameter derived by fitting the Near-Earth Asteroid Thermal Model developed by Harris, 1998, which was used in the analysis of WISE thermal-infrared flux data). Basic taxonomic types are shown as bullets, with M-types being asteroids with possible high metallicity. Higher values of $\eta$ imply lower temperatures on the sunward side of the observed asteroids. The peak in $\eta$ values coincides with the location in the plot of M-type asteroids and a peak in the distribution of radar albedos (b). High radar reflectivity is characteristic of surfaces with a high metal content. Therefore the model fitting parameter, $\eta$, can be used as an indicator of metal-rich asteroids (Harris and Drube, 2014).

Modelling work and computer simulations (see Figure 5 for an example of the results) accompanied the data analysis, with the aim of improving our understanding of the internal structure of small NEOs and their response to deflection attempts. 


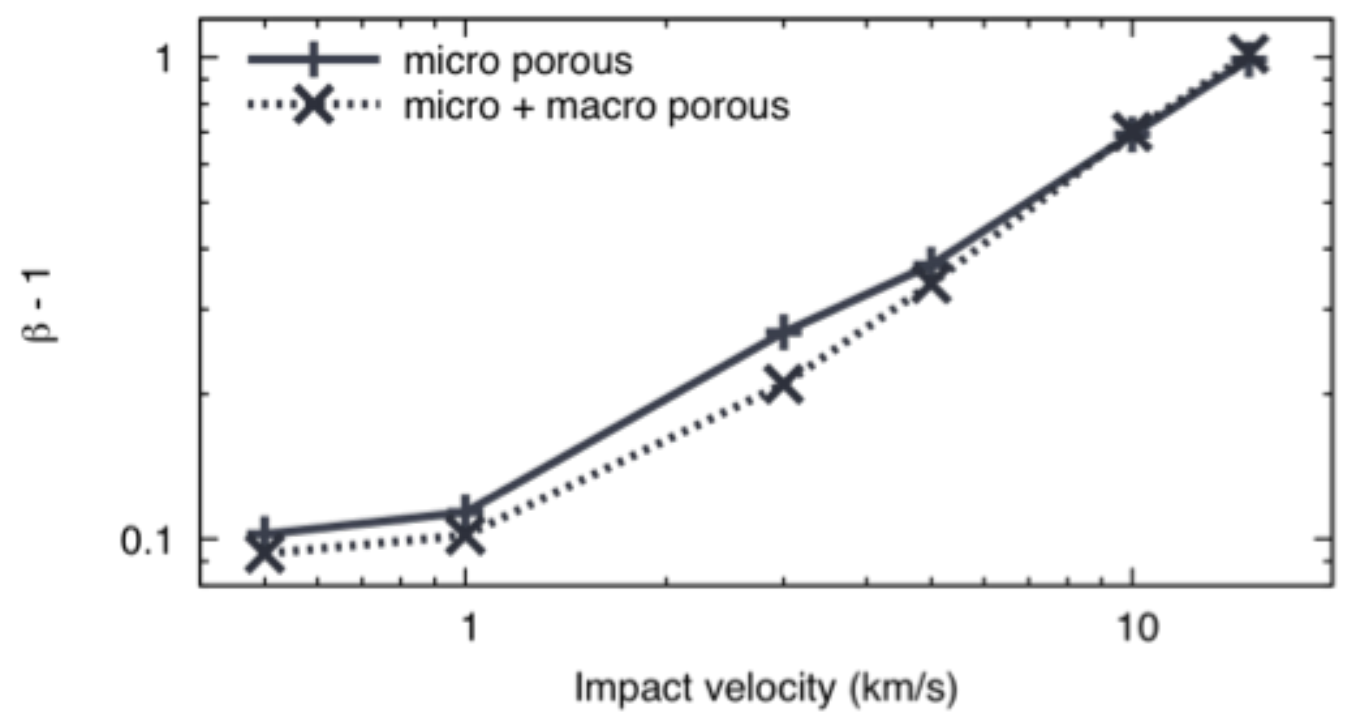

Figure 5: The momentum multiplication factor, $\beta$, versus impact velocity of a projectile. $\beta$ is defined as the ratio between the change in the asteroids momentum after the impact and the momentum of the projectile. Material ejected from the asteroid during the impact will enhance the asteroid's momentum. This graph shows that there is a difference in $\beta$ depending on the porosity of the material. It also shows that at velocities of more than $5 \mathrm{~km} / \mathrm{s}$, the effect of porosity becomes negligible. (Jutzi and Michel, 2014)

In order to validate the models used in the numerical simulations at small scales, impact experiments were carried out in the laboratory using high-velocity light gas guns and targets consisting of asteroid analog materials (Figure 6). Preliminary work carried out within the NEOShield project indicates that the momentum transfer in the case of a porous body is significantly less efficient than in the case of a non-porous body.

Much work remains to be done, however, to enable reliable predictions to be made as to how an asteroid with a particular set of physical parameters would respond to an impacting spacecraft.
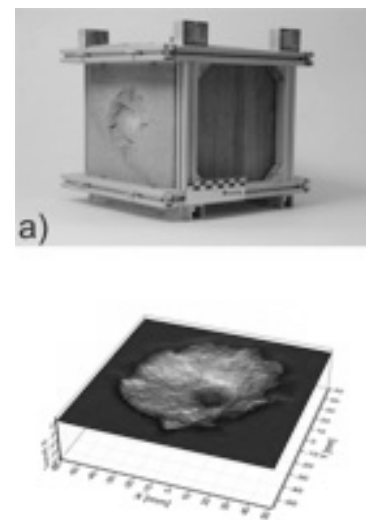
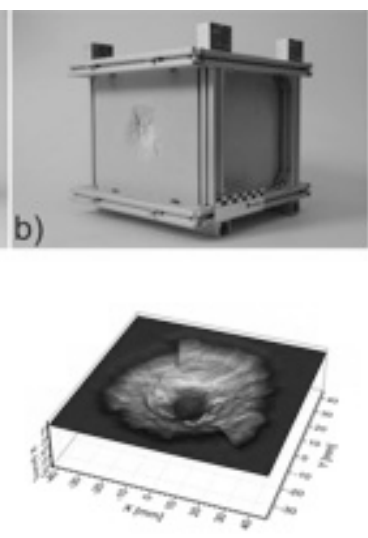
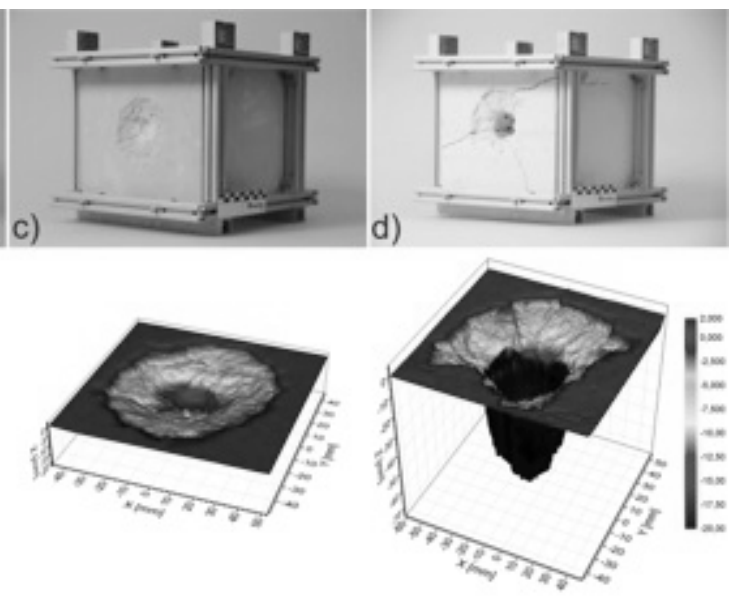

Figure 6: Impact craters formed by hypervelocity impacts into targets with different porosities using a light-gas gun. Porosity rises towards the right. The results show that large craters are created in material with high porosity, but the ejected mass is small, which would reduce the momentum enhancement of the asteroid due to ejecta in a mitigation attempt. Images credit: Fraunhofer Institute for High-Speed Dynamics, Ernst-Mach-Institut, EMI. (Drube et al, 2014) 


\section{Global response campaign roadmap}

Software tools have been developed for NEO impact-risk mitigation within the NEOShield project (Cano et al., 2013). The software tools address the issues of impact risk assessment, and the evaluation of the required deflection and the most efficient deflection strategy. These tools serve as an aid in the selection of the most suitable deflection mission(s) given the circumstances of the potential impact scenario. In the event of a serious impact threat, deciding how to handle the situation would be a major challenge for current political structures, with the danger that considerable time would be wasted before an international whodoes-what agreement could be reached. The United Nations sanctioned two groups in 2014 as part of this endeavour: the International Asteroid Warning Network (IAWN) and the Space Missions Planning Advisory Group (SMPAG). NEOShield partners take part in the activities of the UN-sanctioned groups with a view to setting up a global roadmap to assist decision makers in responding effectively to a confirmed serious impact threat.

\section{Summary}

The NEOShield project was a broad undertaking and at the time of writing results are becoming available in many scientific and technical areas addressed by the project. Results to date include:

- Detailed mission designs based on several types of deflection concept.

- A trade-off analysis of mitigation methods for different asteroid sizes and periods of warning time available for the mitigation attempt.

- A continuously updated prioritized list of asteroids suitable for use as targets in mitigation test missions.

- The discovery of a novel means to identify asteroids with high metal content. Metal content, and associated higher density and robustness would increase the likelihood of an incoming object reaching the ground and causing serious damage. Moreover a threatening object with high metal content and density would require greater effort to deflect.

- Analyses of laboratory experiments in which hypervelocity projectiles are fired into blocks of asteroid analogue materials, showing how the momentum transfer depends on the porosity, impact velocity, material strength, and other parameters

- Computer modelling of high-velocity impacts into asteroids, which show that the momentum enhancement effect due to ejecta is reduced with increased porosity, higher tensile strength, and lower strength against crushing. Results also revealed that the shape of the impacting spacecraft can play an important role..

The article started out with the question "Can we protect our civilization from the next major impact?" While the work of NEOShield has improved our chances, there is still much research needed before we can feel confident in our ability to deflect an asteroid. There are many unknown factors involved in a mitigation attempt. Laboratory work, numerical modelling, and computer simulations are 
essential but not sufficient to enable robust predictions to be made of the outcome of a deflection attempt: It is vitally important to carry out test missions to deflect suitable representative NEO targets before we are forced to apply deflection technology in a hurry in a real impact-prevention scenario.

A successor project to NEOShield, called NEOShield-2, has been granted funding from the European Commission's Horizon 2020 Programme to further develop many aspects of NEOShield research and to increase the readiness level of vital technologies. The work began in March 2015 and will run for 2.5 years. In contrast to NEOShield, NEOShield-2 includes a large work package for observational work on mitigation-relevant NEO targets and associated data reduction and analysis.

\section{Acknowledgments}

The research leading to these results has received funding from the European Union's Seventh Framework Programme (FP7/2007-2013) under grant agreement $\mathrm{n}^{\circ} 282703$ (NEOShield).

\section{References}

Cano, J. L., Bellei, G., and Martin, J. (2013) Integrated end-to-end NEO threat mitigation software suite. Proc. 64th International Astronautical Congress, Beijing, China, 23-27 Sept, 2013 (IAC-13/B5/2/8)

Chapuy, M., Vernis, P., Despré, N., and Capolupo, F. (2014) NEOShield project: GNC design and performance of a liquid-fuelled asteroid kinetic impactor. GNC 2014: 9th International ESA Conf. on Guidance, Navigation, and Control Systems, Porto, Portugal, 2-6 June, 2014

Drube, L., A. W. Harris, T. Hoerth, P. Michel, D. Perna, F. Schäfer, (2014), NEOShield - A global approach to near-Earth object impact threat mitigation, Handbook of Cosmic Hazards and Planetary Defense, ed. J. Pelton, F. Allahdadi, Springer, in production. DOI: 10.1007/978-3-319-03952-7_61

Eggl, S., Ivantsov, A., Hestroffer, D., Perna, D., Bancelin, D, and Thuillot, W. (2013) High precision astrometry in asteroid mitigation - the NEOShield perspective. Proc. Société Française d'Astronomie et a'Astrophysique (SF2A) 2013, Montpellier, France, 4-7 June, 2013

Harris, A. W. and Drube, L., (2014), How to find metal-rich asteroids, Astrophysical Journal Letters, 785, L4, doi:10.1088/2041-8205/785/1/L4

Harris, A. W.; Barucci, M. A.; Cano, J. L.; Fitzsimmons, A.; Fulchignoni, M.; Green, S. F.; Hestroffer, D.; Lappas, V.; Lork, W.; Michel, P.; Morrison, D.; Payson, D. and Schäfer, F., (2013), The European Union funded NEOShield project: a global approach to near-Earth object impact threat mitigation. Acta Astronautica, 90(1) pp. 80-84. doi:10.1016/j.actaastro.2012.08.026 
Harris, A. W., (1998), A thermal model for near-Earth asteroids, Icarus, 131, 291301. doi:10.1006/icar.1997.5865

Jutzi, M., P. Michel, (2014), Hypervelocity impacts on asteroids and momentum transfer I. Numerical simulations using porous targets, Icarus 229, 247-253. DOI:10.1016/j.icarus.2013.11.020

Meshcheryakov, S. A. and Lipnitskii, Y. M. (2015) Estimated efficiency of the deflection of a dangerous space object using an explosion or impact. Technical Physics, 60, 26-30. DOI: 10.1134/S1063784215010181

Ummen, N. and Lappas, V. (2014) Polyhedron tracking and gravity tractor asteroid deflection. Acta Astronautica, 104, 106-124. DOI:

10.1016/j.actaastro.2014.07.024 